Supporting Information for:

\title{
Supported Ni-Au colloid precursors for active, selective, and stable alkyne partial hydrogenation catalysts
}

James E. Bruno, ${ }^{[a]}$ Nicolas S. Dwarica, ${ }^{[a]}$ Todd N. Whittaker,,${ }^{[a]}$ Emily R. Hand, ${ }^{[a]}$ Clemente S. Guzman IV, ${ }^{[a]}$ Anish Dasgupta, ${ }^{[b]}$ Zhifeng Chen, ${ }^{[b]}$ Robert M. Rioux, ${ }^{[b],[c]}$ and Bert D. Chandler ${ }^{*[a],[d]}$

[a] Department of Chemistry, Trinity University One Trinity Place, San Antonio, Texas 78240, United States.

[b] Department of Chemical Engineering, The Pennsylvania State University, University Park, Pennsylvania 16802, United States

[c] Department of Chemistry, The Pennsylvania State University, University Park, Pennsylvania 16802, United States

[d] Laboratorium für Organische Chemie and Laboratorium für Anorganische Chemie, ETH Zürich, $\mathrm{CH}-8093$ Zurich, Switzerland

*To whom correspondence should be addressed: Email: bert.chandler@trinity.edu 


\section{Table of Contents}

S-3 Figure S1. STEM images for c-Ni NPs from Table 1.

S-4 Figure S2. Particle size distribution histograms for (A) 1.9 wt. \% c-Ni4Au, (B) 3.8 wt. \% c-Ni3Au, and (C) 4.4 wt. \% c-Ni3Au.

S-5 Figure S3. Additional EDS images for $\mathrm{Ni}_{2} \mathrm{Au} / \mathrm{Al}_{2} \mathrm{O}_{3}$.

S-6 Figure S4. Additional EDS images for $\mathrm{Ni}_{3} \mathrm{Au}_{\mathrm{A}} \mathrm{Al}_{2} \mathrm{O}_{3}$.

S-7 Figure S5. Additional EDS images for $\mathrm{Ni} 4 \mathrm{Au} / \mathrm{Al}_{2} \mathrm{O}_{3}$.

S-8 Figure S6. IR spectra of $\mathrm{c}-\mathrm{Ni}_{2} / \mathrm{Al}_{2} \mathrm{O}_{3}$ before and after ligand removal treatment. Table S1. Characterization data for $\mathrm{Ni}, \mathrm{Au}$, and c-NiAu catalysts

S-9 Figure S7. Light-off curves for commercial monometallic catalysts. Table S2. Kinetic parameters for commercial monometallic catalysts.

Figure S8. Full catalyst performance FOM plots for monometallic catalysts.

S-10 Figure S9. 1-octene yield plots for c-Au/Al $\mathrm{O}_{3}, \mathrm{c}-\mathrm{Au} / \mathrm{TiO}$, and c-Au/SiO 2 . Figure S10. XRD data for $0.2 \mathrm{wt}$ \% $\mathrm{Au} / \mathrm{NiO}$.

S-11 Figure S11. Hydrogen chemisorption data $\left(30-120^{\circ} \mathrm{C}\right)$ on c-Ni.

S-12 Figure S12. Hydrogen chemisorption data $\left(30-120^{\circ} \mathrm{C}\right)$ on $\mathrm{Ni} / \mathrm{Al}_{2} \mathrm{O}_{3}$.

S-13 Figure S13. Determination of strong $\mathrm{H}_{2}$ adsorption thermodynamics on $\mathrm{Ni} / \mathrm{SiO}_{2}$.

S-14 Figure S14. Van't Hoff plots for total $\mathrm{H}_{2}$ adsorption. 

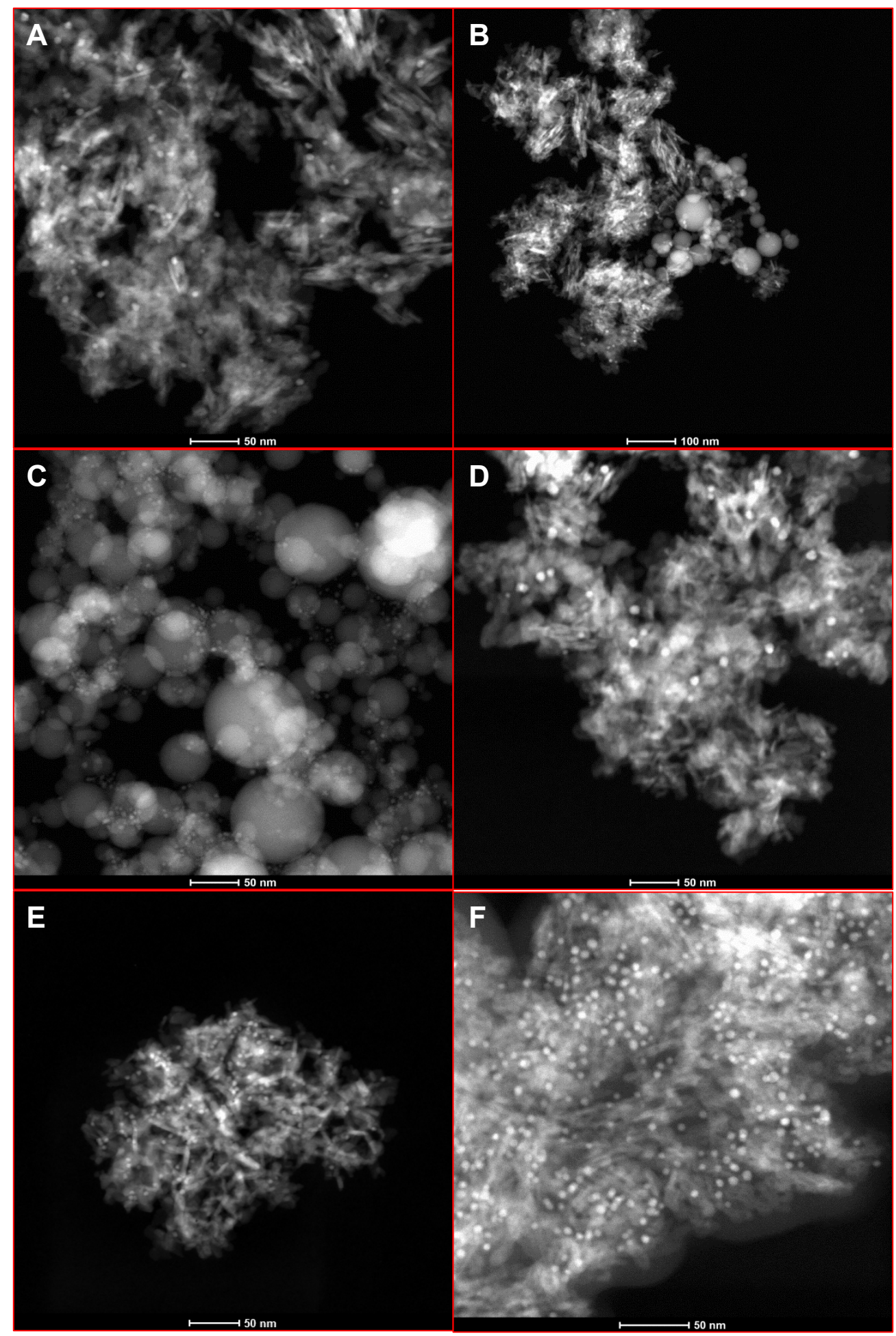

Figure S1. STEM images for c-Ni NPs from Table 1 (letters correspond to Batch). 

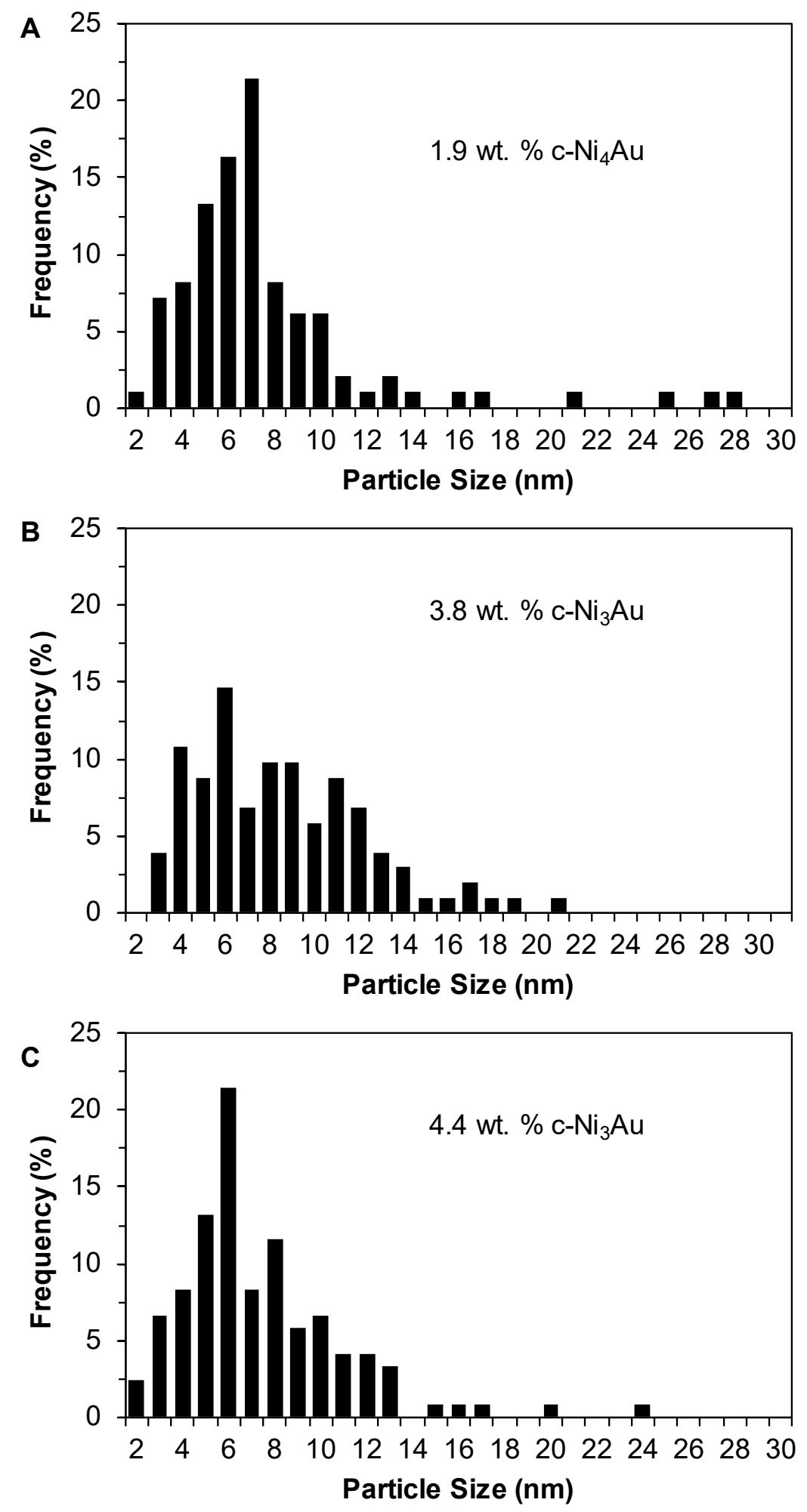

Figure S2. Particle size distribution histograms for (A) 1.9 wt. \% c-Ni4Au, (B) 3.8 wt. \% c$\mathrm{Ni}_{3} \mathrm{Au}$, and (C) 4.4 wt. \% c-Ni3Au. Average particle size diameters are listed in Table 2 of the main text. TEM images are in Figure 9 of the main text. 

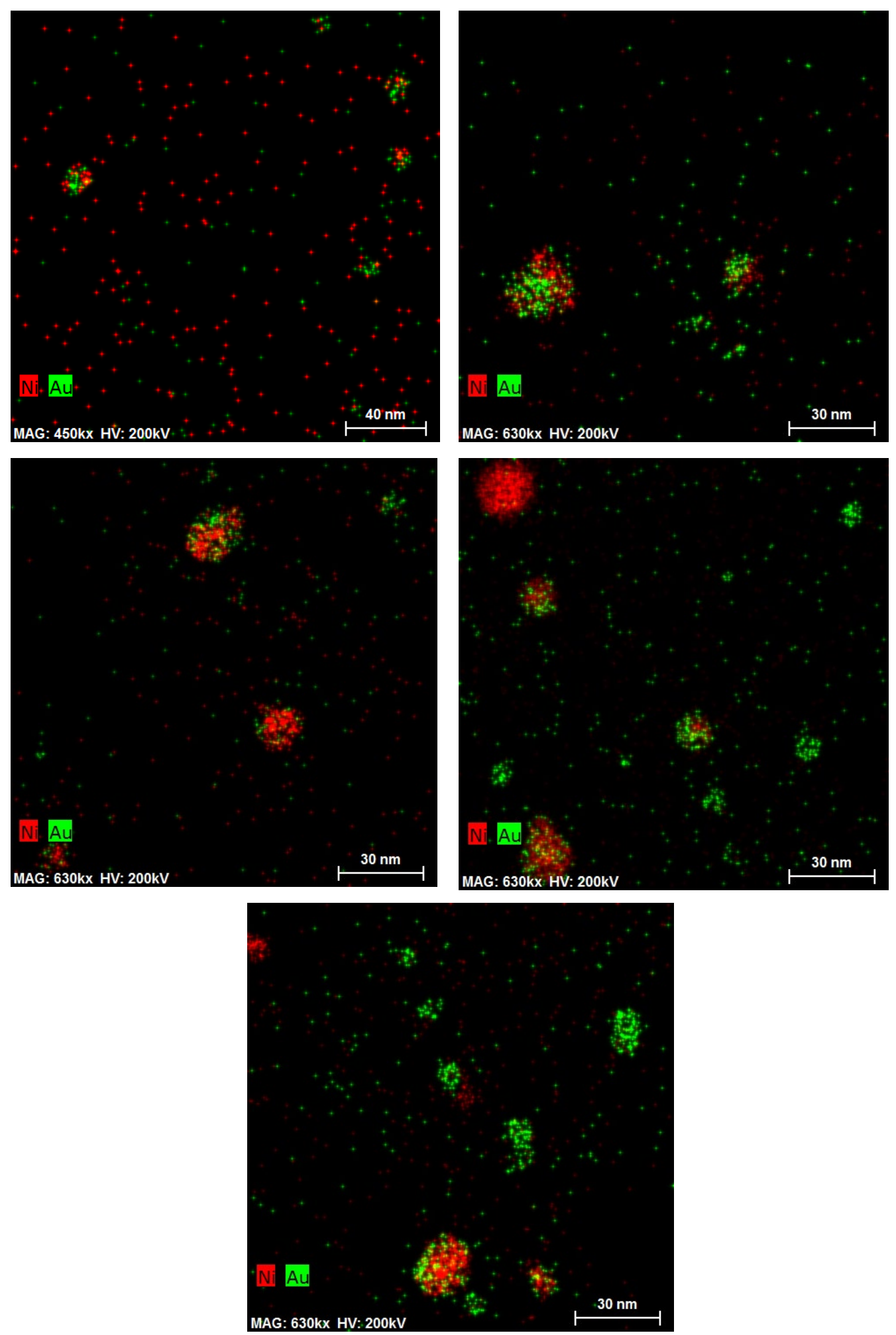

Figure S3. Additional EDS images for $\mathrm{Ni}_{2} \mathrm{Au} / \mathrm{Al}_{2} \mathrm{O}_{3}$. 

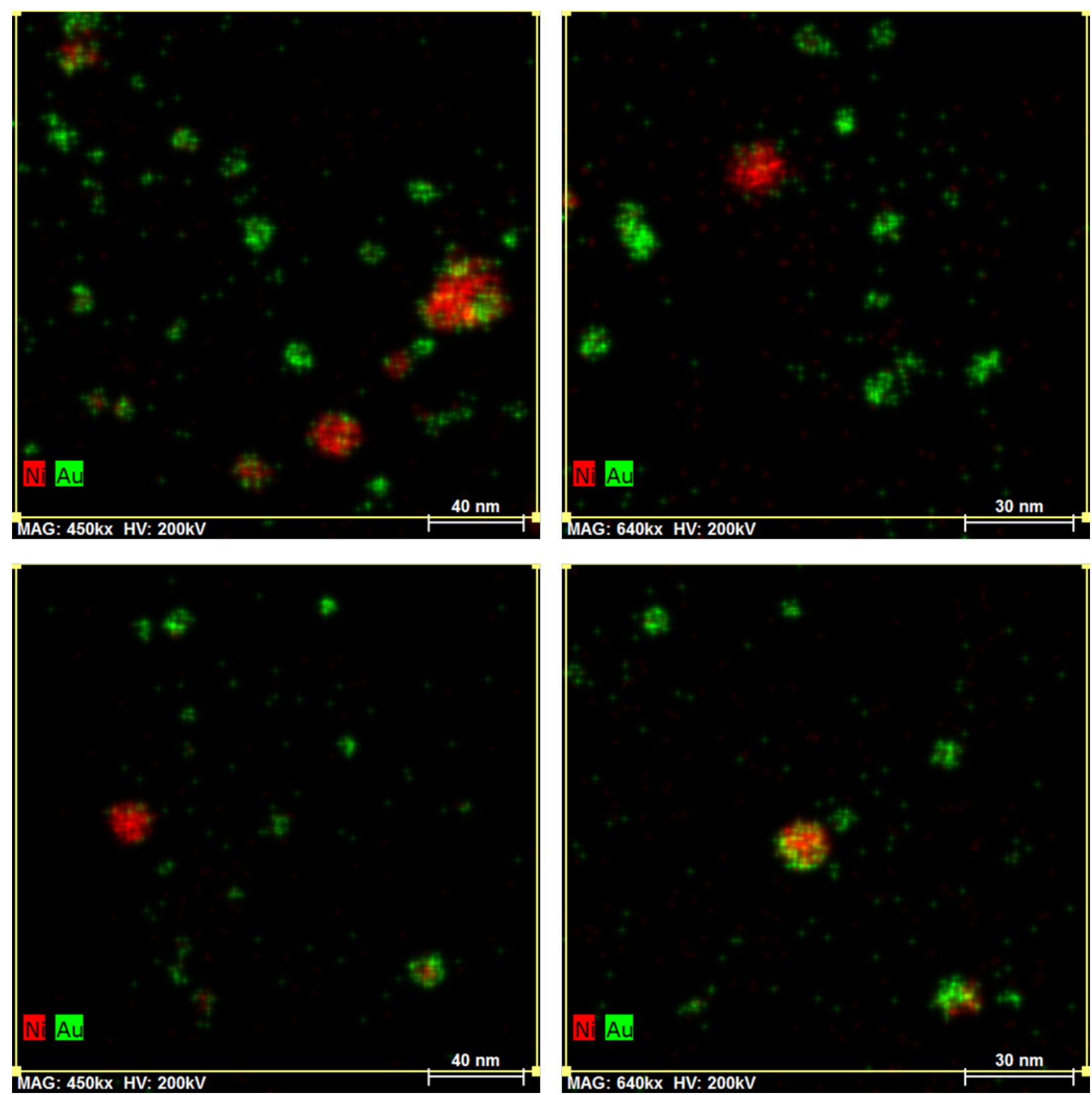

Figure S4. Additional EDS images for $\mathrm{Ni}_{3} \mathrm{Au}_{\mathrm{u}} \mathrm{Al}_{2} \mathrm{O}_{3}$. 

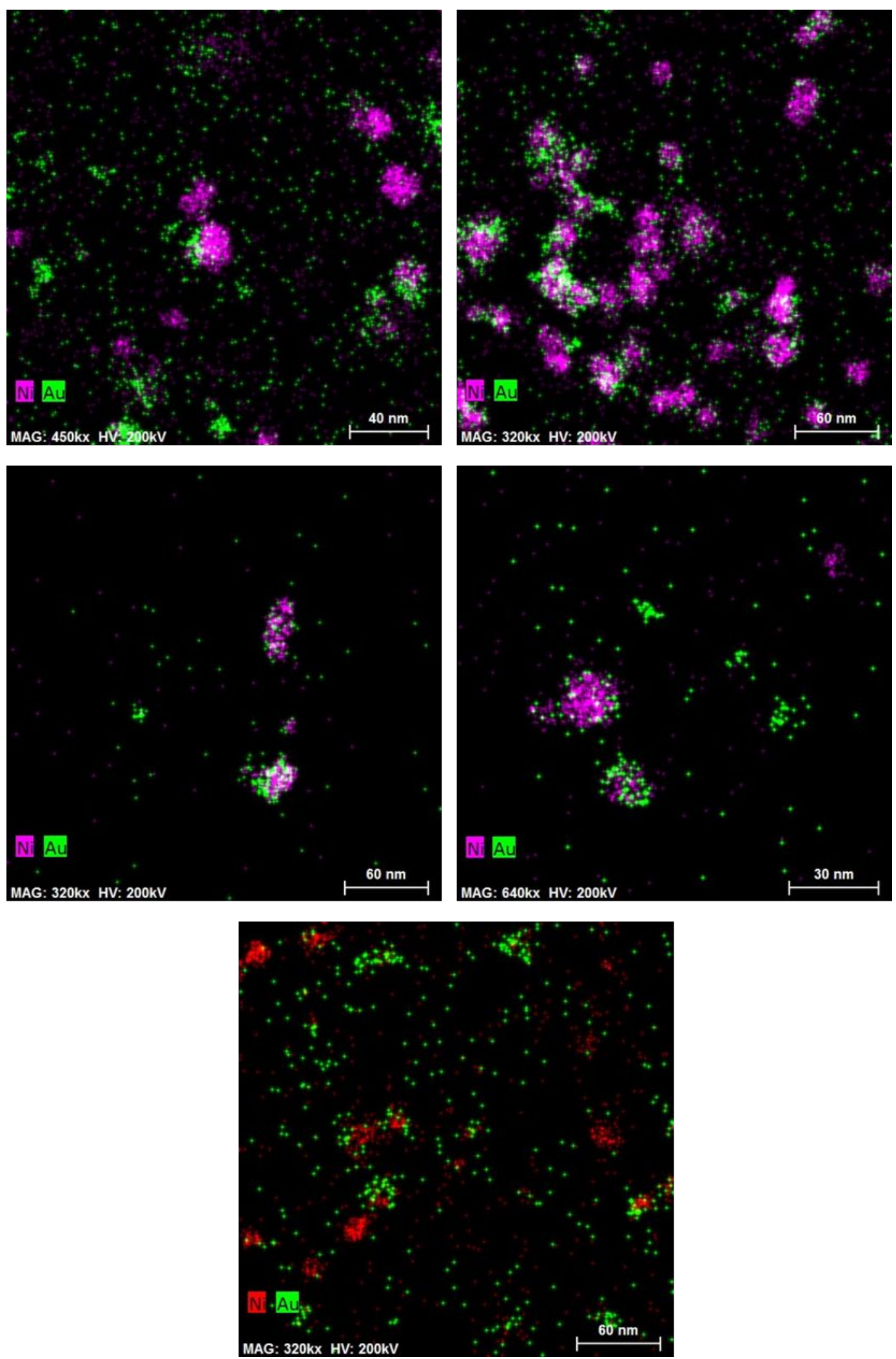

Figure S5. Additional EDS images for $\mathrm{Ni} 4 \mathrm{Au} / \mathrm{Al}_{2} \mathrm{O}_{3}$. 


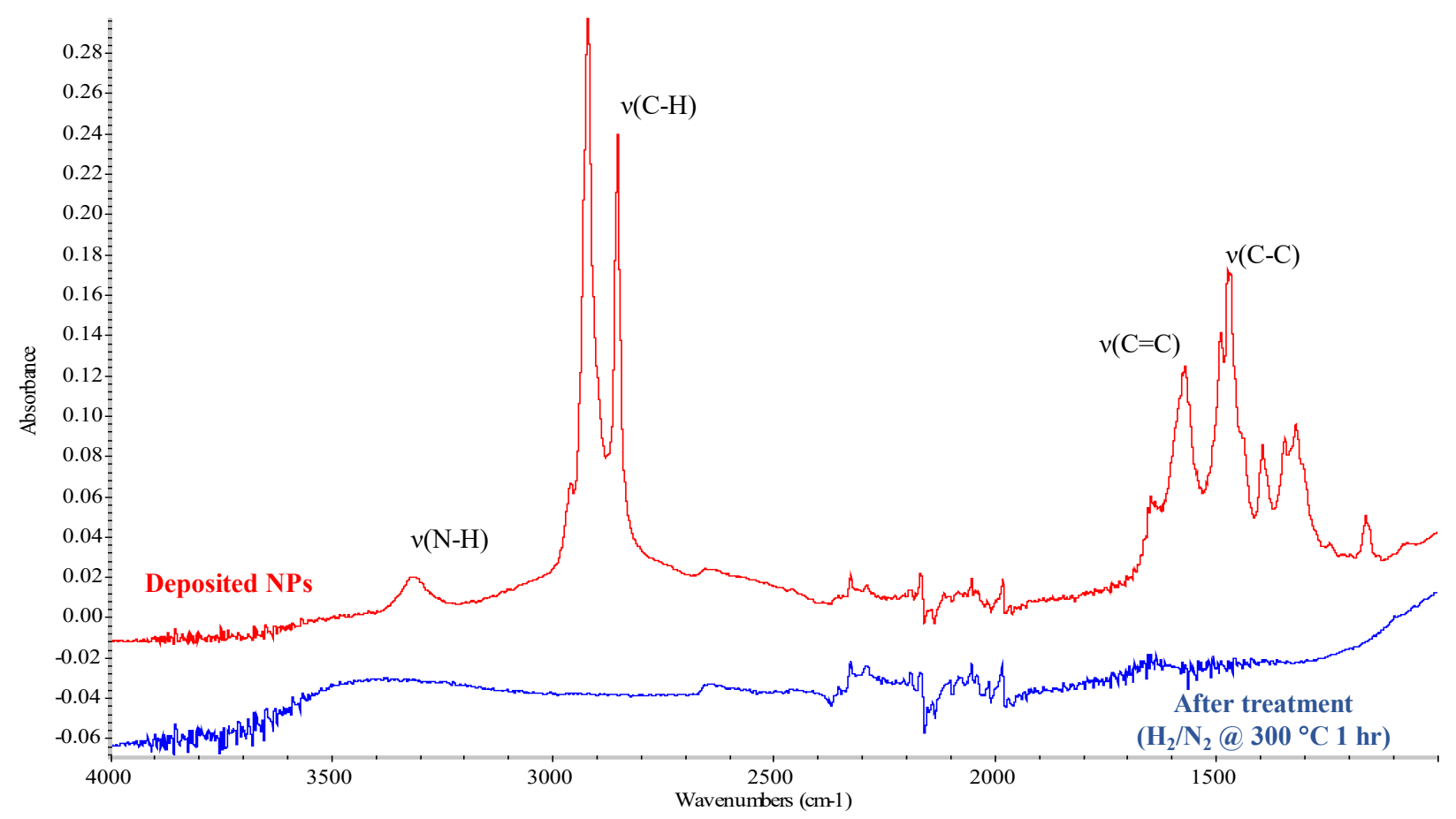

Figure S6. IR spectra of $\mathrm{c}-\mathrm{Ni} / \mathrm{Al}_{2} \mathrm{O}_{3}$ before and after ligand removal treatment.

Table S1: Characterization data for $\mathrm{Ni}, \mathrm{Au}$, and c-NiAu catalysts

\begin{tabular}{|c|c|c|c|}
\hline Catalyst & Ni wt. \% & Au wt. \% & Surface Area $\left(\mathrm{m}^{2} / \mathrm{g}\right)$ \\
\hline STREM Au/Al $\mathrm{O}_{3}$ & 0 & 0.8 & 240 \\
\hline $\mathrm{NIH} \mathrm{Ni/AI}{ }_{2} \mathrm{O}_{3}$ & 17 & 0 & 150 \\
\hline $\mathrm{c}-\mathrm{Au} / \mathrm{Al}_{2} \mathrm{O}_{3}$ & 0 & 1.3 & 80 \\
\hline $\mathrm{c}-\mathrm{Ni} / \mathrm{Al}_{2} \mathrm{O}_{3}$ & 1.4 & 0 & 150 \\
\hline 1.9 wt. $\% \mathrm{c}-\mathrm{NiAu} / \mathrm{Al}_{2} \mathrm{O}_{3}$ & 1.0 & 0.9 & 130 \\
\hline 3.8 wt. $\% \mathrm{c}-\mathrm{NiAu} / \mathrm{Al}_{2} \mathrm{O}_{3}$ & 1.6 & 2.2 & 140 \\
\hline 4.4 wt. $\% \mathrm{c}-\mathrm{NiAu} / \mathrm{Al}_{2} \mathrm{O}_{3}$ & 2.1 & 2.3 & 130 \\
\hline $\mathrm{Al}_{2} \mathrm{O}_{3}$ Support & - & - & 140 \\
\hline
\end{tabular}



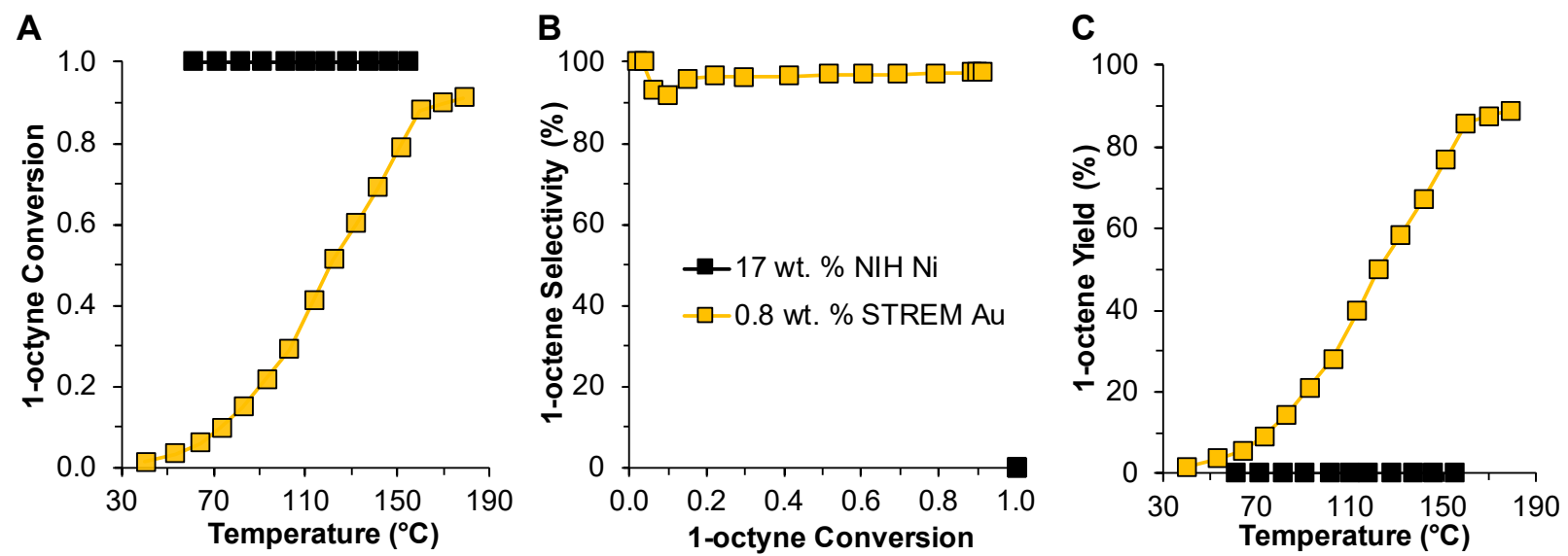

Figure S7. Light-off curves (A), 1-octene selectivity (B), and 1-octene yield (C) for commercial monometallic catalysts. Conditions: $\sim 5 \mathrm{mg}$ catalyst, $50 \mathrm{~mL} / \mathrm{min} \mathrm{H}_{2}, 20 \mathrm{~Pa} 1$ octyne, GHSV $=240,000 \mathrm{~h}^{-1}$.

Table S2: Kinetic parameters for commercial monometallic catalysts.

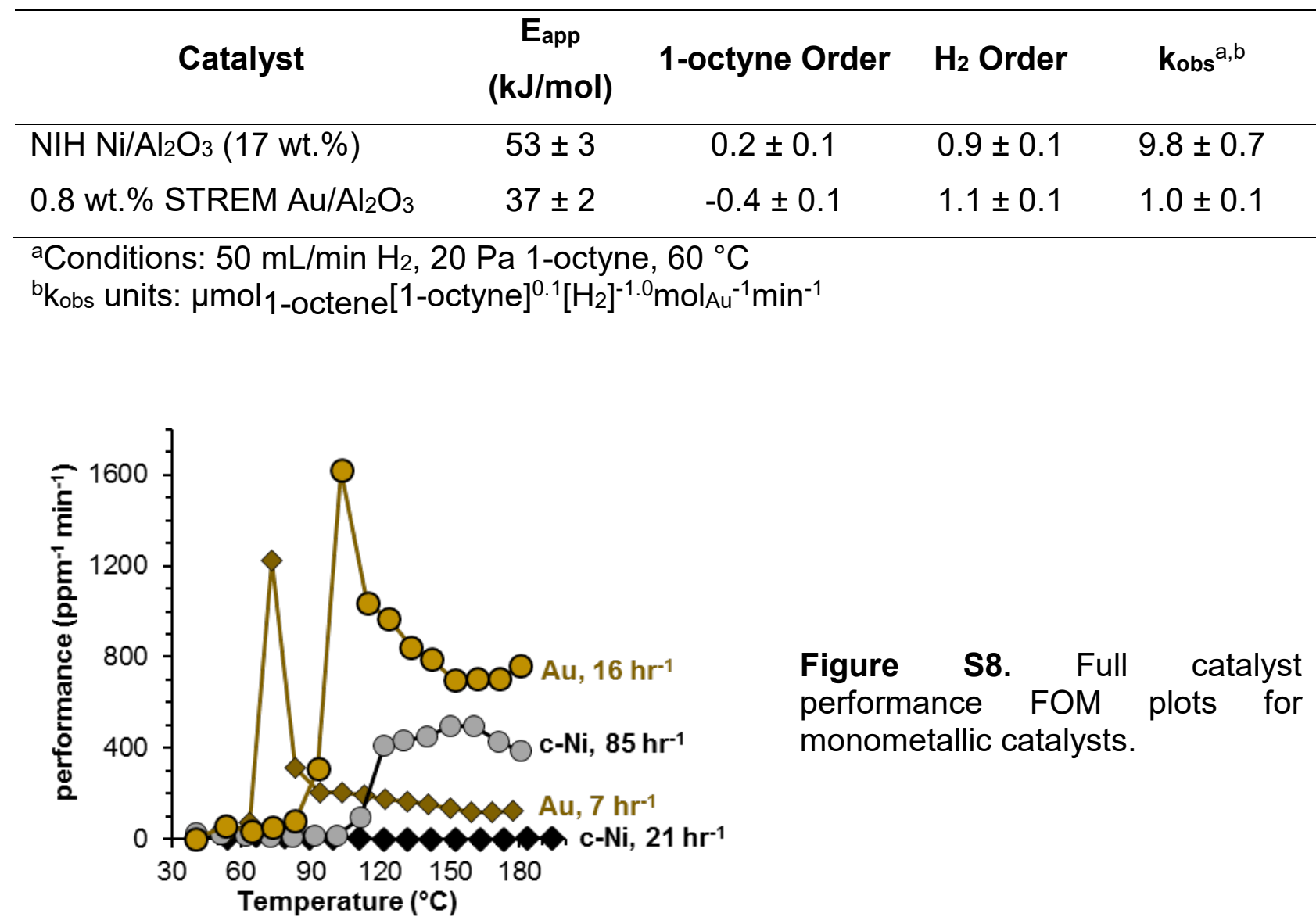



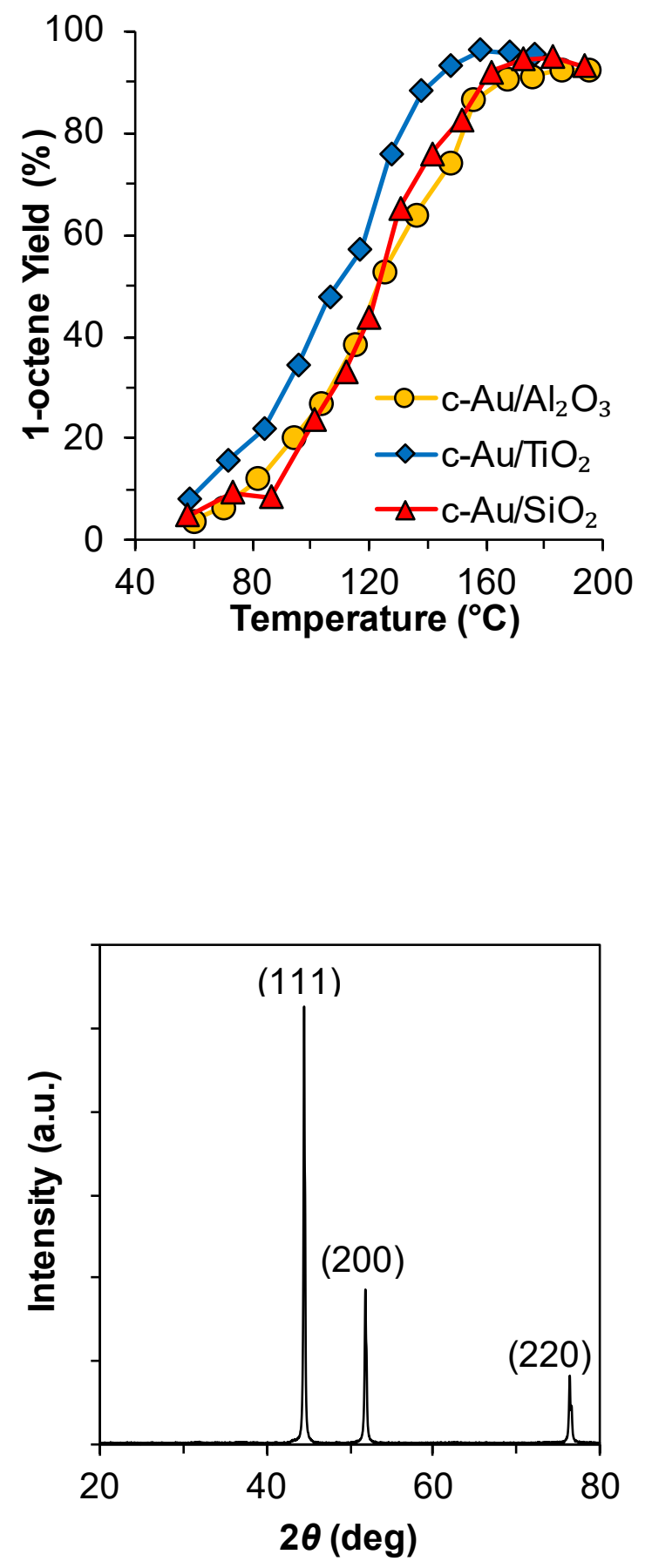

Figure S9. 1-octene yield plots for c$\mathrm{Au} / \mathrm{Al}_{2} \mathrm{O}_{3}, \mathrm{c}-\mathrm{Au} / \mathrm{TiO}$, and $\mathrm{c}-\mathrm{Au} / \mathrm{SiO}_{2}$. Yield was calculated with conversion and selectivity data. Conditions: $50 \mathrm{~mL} / \mathrm{min} \mathrm{H}_{2}, 20 \mathrm{~Pa}$ 1octyne, $\sim 7 \mathrm{mg}$ catalyst, GHSV $=170,000 \mathrm{~h}^{-1}$.
Figure S10. XRD data for 0.2 wt. \% Au/NiO. The three peaks correspond with $\mathrm{Ni}(0)$ [PDF(04-003-7363)]. This diffractogram was measured using Co $\mathrm{K}_{a}$ radiation; while the diffractograms in the main text used $\mathrm{Cu} \mathrm{Ka}_{\alpha}$ radiation. 

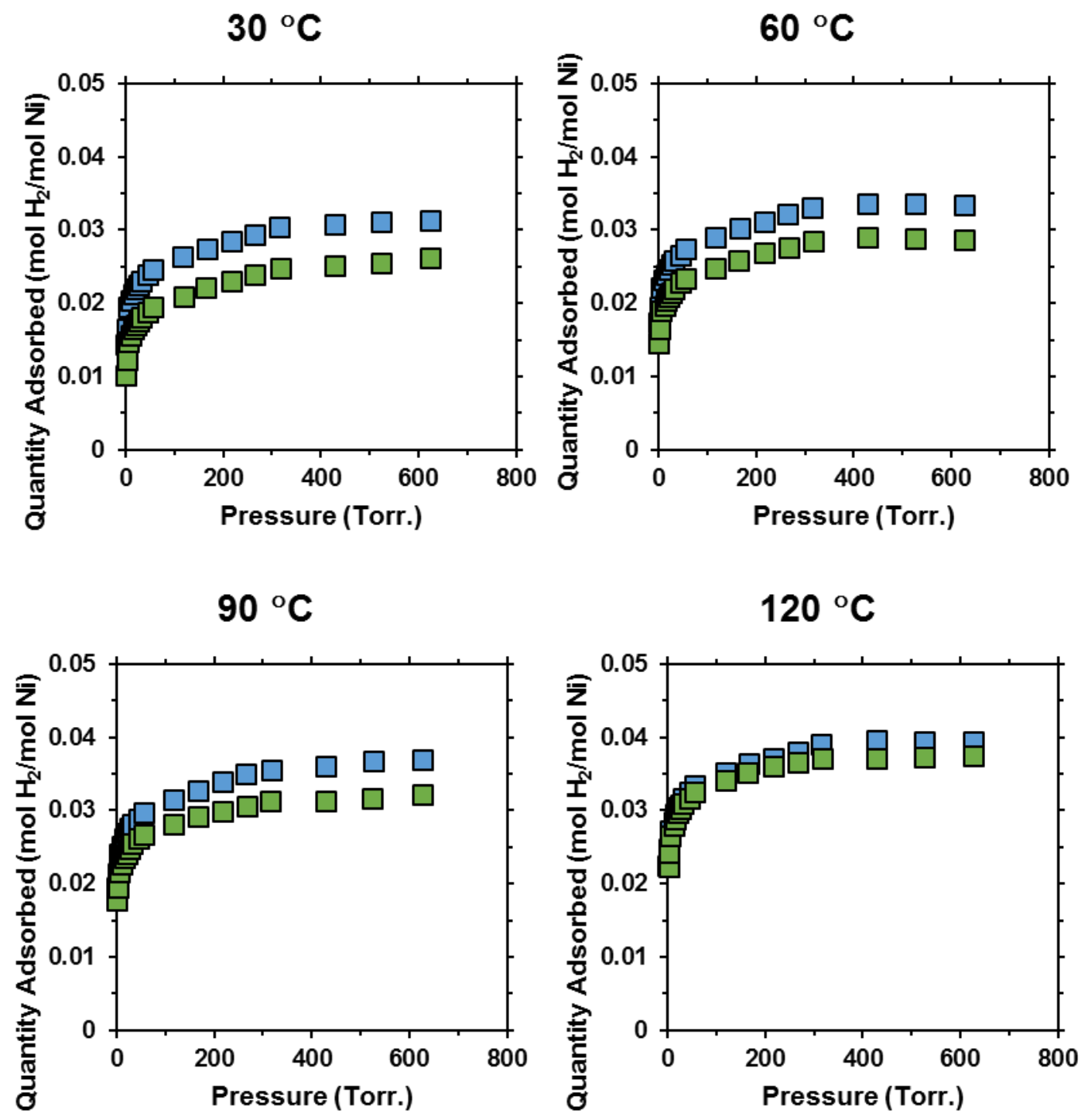

Figure S11. Hydrogen chemisorption data $\left(30-120^{\circ} \mathrm{C}\right)$ on c-Ni. 

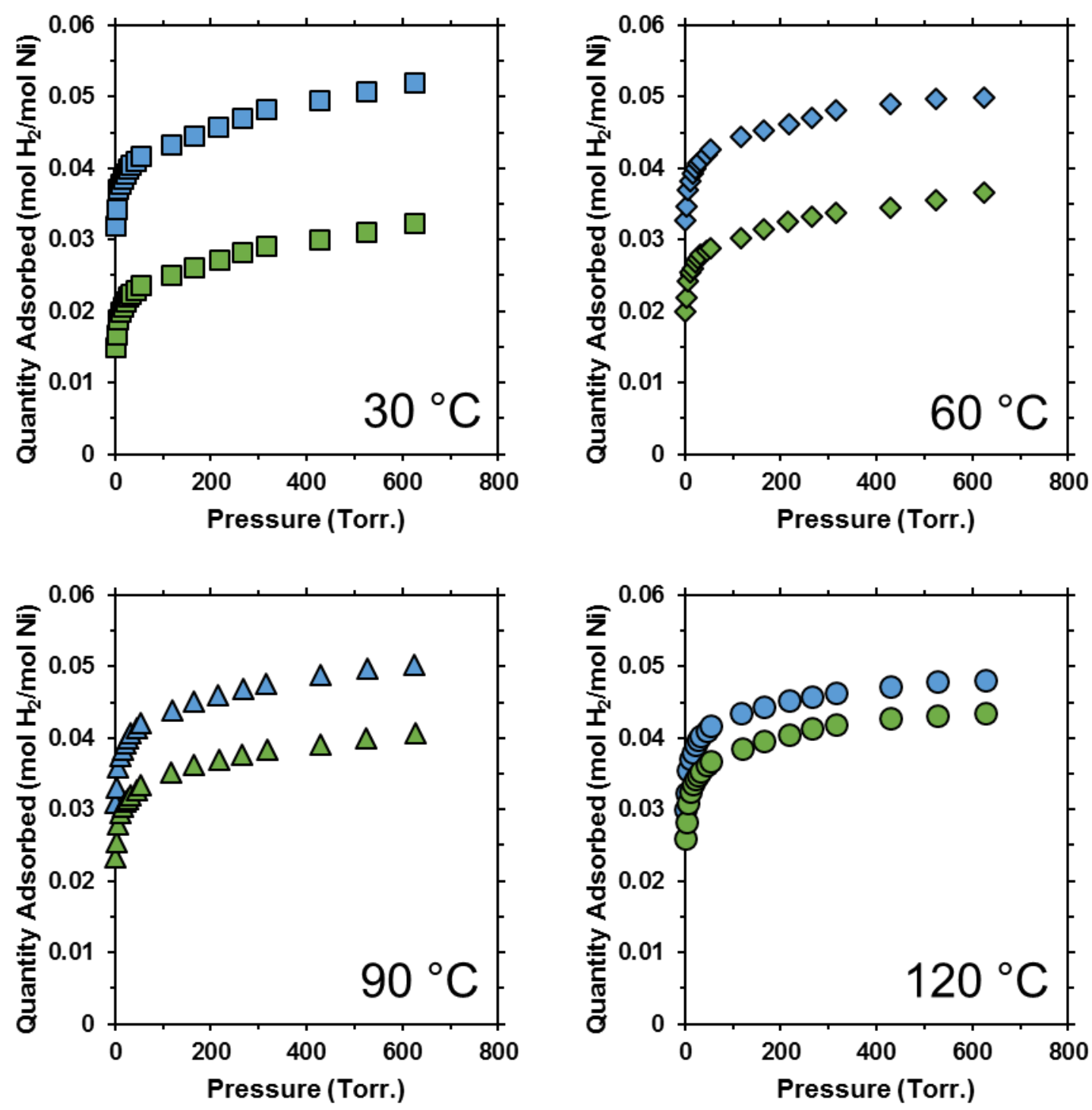

Figure S12. Hydrogen chemisorption data on $\mathrm{Ni} / \mathrm{SiO}_{2}$. 
Method 1
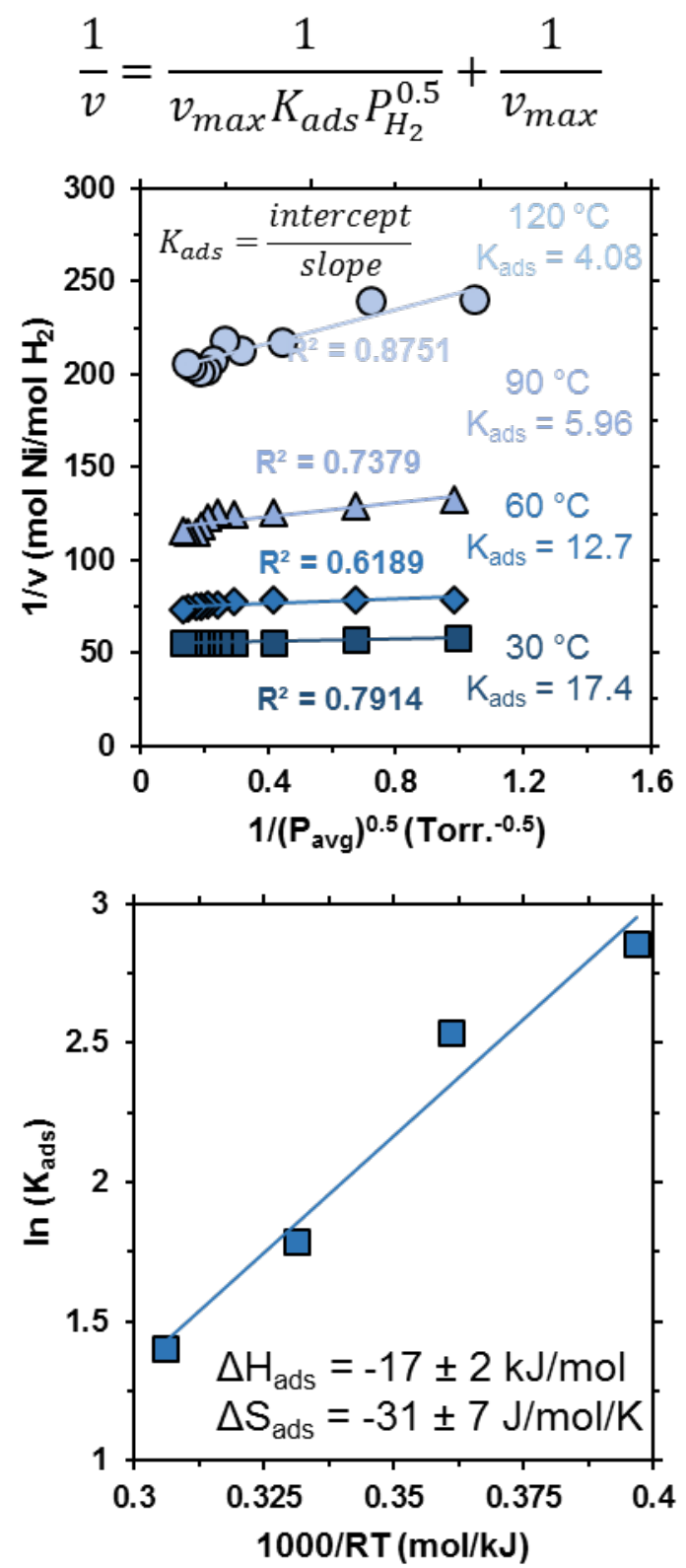

Method 2

$\frac{P_{H_{2}}^{0.5}}{v}=\frac{1}{v_{\max } K_{a d s}}+\frac{P_{H_{2}}^{0.5}}{v_{\max }}$
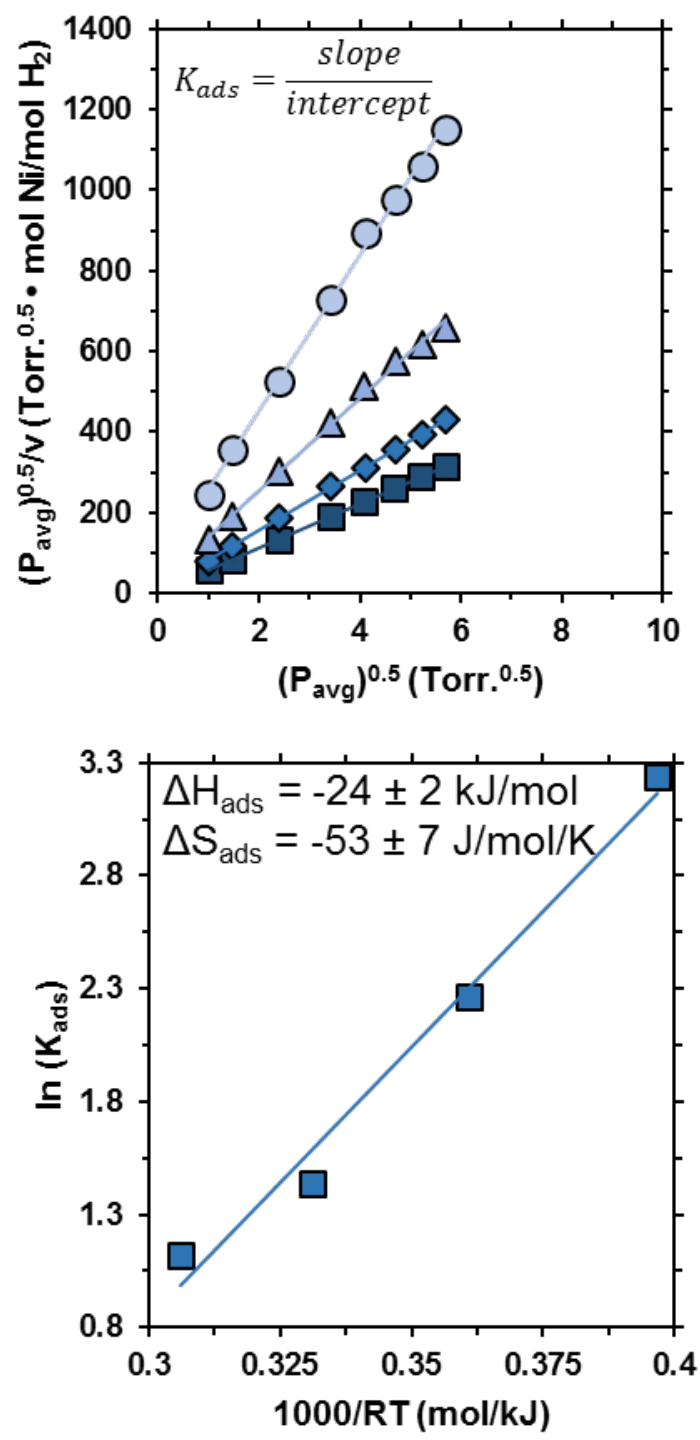

Figure S13. Determination of strong $\mathrm{H}_{2}$ adsorption thermodynamics on $\mathrm{Ni} / \mathrm{SiO}_{2}$. 


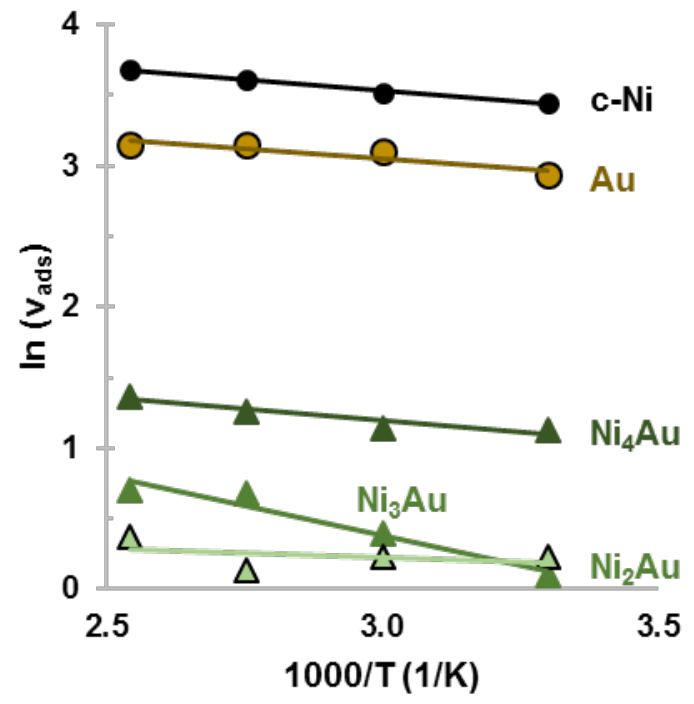

Figure S14. Van't Hoff plots for total $\mathrm{H}_{2}$ adsorption. 